\title{
Estimation of the Loss and Risk Functions of Parameter of Maxwell Distribution
}

\author{
Guobing Fan \\ Department of Basic Subjects, Hunan University of Finance and Economics, Changsha, China
}

Email address:

fanguobingmath@163.com

\section{To cite this article:}

Guobing Fan. Estimation of the Loss and Risk Functions of Parameter of Maxwell Distribution. Science Journal of Applied Mathematics and Statistics. Vol. 4, No. 4, 2016, pp. 129-133. doi: 10.11648/j.sjams.20160404.12

Received: May 21, 2016; Accepted: June 6, 2016; Published: June 29, 2016

\begin{abstract}
In statistical decision-making, when Bayes estimator is used as the unknown parameter's estimation, there often exists certain loss. Then the aim of this paper is to study the Bayes estimation for the loss and risk functions of parameter of Maxwell distribution under Rukhin's loss function. Bayes estimator is derived on the basis of the inverse gamma prior distribution under squared error loss function. Then Bayes estimators of loss and risk function are obtained, respectively. Finally, the conditions of Bayes estimators being conservative are also derived.
\end{abstract}

Keywords: Bayes Estimator, Loss Function, Risk Function, Maxwell Distribution

\section{Introduction}

In statistical decision making process, when an estimator $d=\delta(x)$ is used as the unknown parameters estimation, it can bring certain loss. Here we note the loss function is $w(\theta, \delta)$, then $w(\theta, \delta)$ is also not a crisp result due to it is also unknown. So we need estimate the loss function $w(\theta, \delta)$. Classical decision theory advocates making some decision $d=\delta(x)$, where $x$ is the observation, with frequentist risk $R(\theta, \delta)$. This approach has been often criticized because it implicitly assumes that $R(\theta, \delta)$ is a good measure of accuracy of the procedure used (or of a measure of conclusiveness). In 1988, Rukhin proposed a loss function [1]

$$
L(\theta ; \delta, \gamma)=w(\theta, \delta) \gamma^{-\frac{1}{2}}+\gamma^{\frac{1}{2}},
$$

Here $\gamma$ is an estimator of loss $w(\theta, \delta)$. The loss function $L(\theta ; \delta, \gamma)$ combines the non-negative decision loss $w(\theta, \delta)$ with the accuracy of $\delta$. This loss function is a very convenient tool in the problem of simultaneous "decisionprecision" reporting. The corresponding risk function has frequentist interpretability in terms of long-run frequencies.

Loss and risk functions are important parts in statistical inference. Many valuable results are obtained under different loss functions, and the performances of estimators are compared under risk function [2-6]. For the estimations of loss and risk functions of parameter of binomial distribution, Rukhin [1] attained the Bayes estimators under the loss $L(\theta ; \delta, \gamma)$, and he also gave its properties; Later, many authors studied estimation of loss and risk functions of paramerers for various lifetime distributions models, such as, Poisson distribution and exponential distribution [7], normal and lognormal distribution [8, 9], Rayleigh distribution [10]. The conditions of conservative properties are also discussed in these references.

The Maxwell distribution was first introduced by Maxwell in 1860 as a lifetime distribution model, and since then, the study and application of Maxwell distribution have been received great attention. Tyagi and Bhattacharya [11] firstly considered Maxwell distribution as a life time model, and they obtained the minimum variance unbiased estimator (UMVUE) and Bayes estimator of the parameter and reliability of this distribution. Chaturvedi and Rani [12] studied Bayesian reliability estimation of the generalized Maxwell failure distribution. Podder and Roy [13] studied the estimation of the parameter of this distribution under modified linear exponential loss (MLINEX) function. Bekker and Roux [14] discussed the maximum likelihood estimator (MLE), Bayes estimators of the truncated first moment and hazard function of the Maxwell distribution. Dey and Maiti 
[15] derived Bayes estimators of Maxwell distribution by considering non-informative and conjugate prior distributions under three loss functions, namely, quadratic loss function, squared-log error loss function and MLINEX function.

Let $X$ be a random variable distributed with Maxwell distribution with the following probability density function (pdf) (Krishna and Malik [16]):

$$
f(x ; \theta)=\frac{4}{\sqrt{\pi}} \theta^{-\frac{3}{2}} x^{2} e^{-\frac{x^{2}}{\theta}}, \quad x>0, \theta>0
$$

Here, $\theta$ is the unknown parameter.

The purpose of this paper is to study Bayes estimation of the loss and risk functions of Maxwell distribution on the basis of the inverse gamma prior distribution under squared error loss function. Some conservative of Bayes estimators will also be obtained.

\section{Preliminary Knowledge}

This section will give some preliminary knowledge for the further discussion of the estimation of loss and risk function of Maxwell distribution (2).

For an estimator, risk function is often used as a method to measure the performance of this estimator. The risk function is still a function of unknown parameter, thus it is also unknown. Thus it has been often criticized when we use the risk function to measure the performance of estimators. Rukhin's loss function (1) can overcome this shortcoming, and it has the following properties:

(i) For a fixed $d=\delta(x), L(\theta ; \delta, \gamma)$ gets its (unique) minimum in $\gamma$ at the point $\gamma_{\min }=w(\theta, \delta)$. A convenient convention is to put $\gamma_{\min }=0$ when $w(\theta, \delta)=0$.

(ii) For any fixed $\gamma \geq 0$, the loss function $L(\theta ; \delta, \gamma)$ is just a linear transformation of $w(\theta, \delta)$, so that the Bayes estimator $\delta_{B}(X)$ of $\theta$ for $L(\theta ; \delta, \gamma)$ is the Bayesian procedure for $w(\theta, \delta)$. The Bayes estimator $\gamma_{B}(X)$ of accuracy is

$$
\gamma_{B}(x)=E\left[w\left(\theta, \delta_{B}\right) \mid X\right]
$$

Definition1 Supposed $\gamma(X)$ is estimator of $w(\theta, \delta)$ with unknown parameter $\theta, \gamma(X)$ is called a conservative estimator, if it satisfies: $E_{\theta}(\gamma(X)) \geq R(\theta, \delta)=E_{\theta}[w(\theta, \delta)]$.

Let $X=\left(X_{1}, X_{2}, \ldots, X_{n}\right)$ be a sequence of independent and identically distributed random variables of Maxwell distribution with pdf (2), and $x=\left(x_{1}, x_{2}, \ldots, x_{n}\right)$ is the observation of $X$. The likelihood function of $\theta$ for the given sample observation is

$$
\begin{aligned}
l(\theta ; x) & =\prod_{i=1}^{n} \frac{4}{\sqrt{\pi}} \theta^{-\frac{3}{2}} x_{i}^{2} e^{-\frac{x_{i}^{2}}{\theta}} \\
& =\left(\frac{4}{\sqrt{\pi}}\right)^{n} \cdot \theta^{-\frac{3}{2} n} \cdot\left(\prod_{i=1}^{n} x_{i}^{2}\right) \cdot e^{-\frac{1}{\theta} \sum_{i=1}^{n} x_{i}^{2}} \\
& =K \cdot \theta^{-\frac{3}{2} n} \cdot e^{-\frac{1}{\theta} \sum_{i=1}^{n} x_{i}^{2}}
\end{aligned}
$$

Here $K=\left(\frac{4}{\sqrt{\pi}}\right)^{n} \cdot\left(\prod_{i=1}^{n} x_{i}^{2}\right)$ is a proportionality constant with respect to the parameter $\theta$. And in Bayesian statistics, we usually use $\propto$ to demonstrate the likelihood kernel. That is $l(\theta ; x)$ can also be rewrritten as

$$
l(\theta ; x) \propto \theta^{-\frac{3}{2} n} e^{-\frac{1}{\theta} \sum_{i=1}^{n} x_{i}^{2}} \propto \theta^{-\frac{3}{2} n} e^{-\theta t}
$$

Where $t=\sum_{i=1}^{n} x_{i}^{2}$ is the observation of $T=\sum_{i=1}^{n} X_{i}^{2}$.

By solving $\log$ likelihood equation, the maximum likelihood estimator (MLE) of $\theta$ is easily derived as follows:

$$
\hat{\theta}_{M}=\frac{3 n}{T}
$$

And by Eq. (1), we can also show that $T$ is distributed with Gamma distribution $\Gamma\left(\frac{3}{2} n, \frac{1}{\theta}\right)$, which has the following probability density function:

$$
f_{T}(t ; \theta)=\frac{\theta^{-\frac{3}{2} n}}{\Gamma\left(\frac{3}{2} n\right)} t^{\frac{3}{2} n-1} e^{-\frac{t}{\theta}}, \quad t>0, \theta>0
$$

\section{Bayes Estimation of Loss and Risk Function}

In this section, we shall concern the estimation of the loss and risk function of Maxwell distribution (2). For later use, we consider the Bayes estimation of $\theta$ under the following square error loss function

$$
w(\theta, \delta)=(\theta-\delta)^{2}
$$

Theorm 1 Let $X=\left(X_{1}, X_{2}, \ldots, X_{n}\right)$ be a sequence of independent and identically distributed random variables of Maxwell distribution with pdf (2), and $x=\left(x_{1}, x_{2}, \ldots, x_{n}\right)$ is the observation of $X$. The conjugate prior distribution for $\theta$ is the inverse Gamma distribution, and $\gamma(X)$ is an estimator of $w(\theta, \delta)$, then

(i) Under squared error loss function (8), the Bayes 
ertimator of $\theta$ is:

$$
\delta_{B}=\frac{\beta+T}{\frac{3}{2} n+\alpha-1}
$$

(ii) The Bayes estimator of $w(\theta, \delta)$ based on Rukhin's loss function (1) is:

$$
\gamma_{B}(X)=\frac{(\beta+T)^{2}}{\left(\frac{3}{2} n+\alpha-1\right)^{2}\left(\frac{3}{2} n+\alpha-2\right)}
$$

and

$$
E_{\theta} \gamma_{B}(X)=\frac{\frac{3}{2} n\left(\frac{3}{2} n+1\right) \theta^{2}+3 \beta \theta+\beta^{2}}{\left(\frac{3}{2} n+\alpha-1\right)^{2}\left(\frac{3}{2} n+\alpha-2\right)}
$$

(iii) The risk function of $\delta_{B}$ is:

$$
R\left(\theta, \delta_{B}\right)=\frac{\left[\frac{3}{2} n+(\alpha-1)^{2}\right] \theta^{2}+2 \beta(1-\alpha) \theta+\beta^{2}}{\left(\frac{3}{2} n+\alpha-1\right)^{2}}
$$

Proof

(i) Suppose that the conjugate family of prior distributions for $\theta$ is the inverse Gamma distributions, $I \Gamma(\alpha, \beta)$, with the probability density function:

$$
\pi(\theta \mid \alpha, \beta)=\frac{\beta^{\alpha}}{\Gamma(\alpha)} \theta^{-(\alpha+1)} e^{-\frac{\beta}{\theta}}, \theta>0, \alpha, \beta>0
$$

Then the posterior distribution of $\theta$ is also inverse Gamma distribution $I \Gamma\left(\alpha+\frac{3}{2} n, \beta+T\right)$, Thus

$$
\hat{\delta}_{B}=E(\theta \mid X)=\frac{\beta+T}{\frac{3}{2} n+\alpha-1} ;
$$

(ii) The Bayes estimator of $w(\theta, \delta)$ under the Rukhin's loss function (1) is:

$$
\begin{aligned}
\gamma_{B}(X) & =E\left[w\left(\theta, \delta_{B}\right) \mid X\right] \\
& =E\left[\left(\theta-\delta_{B}(X)\right)^{2} \mid X\right] \\
& =\operatorname{Var}(\theta \mid X) \\
& =\frac{(\beta+T)^{2}}{\left(\frac{3}{2} n+\alpha-1\right)^{2}\left(\frac{3}{2} n+\alpha-2\right)}
\end{aligned}
$$

Thus

$$
\begin{aligned}
E_{\theta} \gamma_{B}(X)= & \frac{1}{\left(\frac{3}{2} n+\alpha-1\right)^{2}\left(\frac{3}{2} n+\alpha-2\right)} E\left[(\beta+T)^{2} \mid X\right) \\
& =\frac{\frac{3}{2} n\left(\frac{3}{2} n+1\right) \theta^{2}+3 \beta n \theta+\beta^{2}}{\left(\frac{3}{2} n+\alpha-1\right)^{2}\left(\frac{3}{2} n+\alpha-2\right)}
\end{aligned}
$$

(iii) iii) The Risk function of $\delta_{B}$ :

$$
\begin{aligned}
R\left(\theta, \delta_{B}\right) & =E_{\theta}\left[W\left(\theta, \delta_{B}\right)\right] \\
& =E_{\theta}\left[\left(\theta-\frac{\beta+T}{\frac{3}{2} n+\alpha-1}\right)^{2}\right] \\
& =\frac{\left[\frac{3}{2} n+(\alpha-1)^{2}\right] \theta^{2}+2 \beta(1-\alpha) \theta+\beta^{2}}{\left(\frac{3}{2} n+\alpha-1\right)^{2}}
\end{aligned}
$$

Because $R(\theta, \delta)=E_{\theta}[W(\theta, \delta)]$ is the mean value of $W(\theta, \delta)$, then we can regard $R(\theta, \delta)$ as an estimator of $W(\theta, \delta)$. But the risk function $R(\theta, \delta)=E_{\theta}[W(\theta, \delta)]$ as the average loss of decision $d=\delta(X)$ is the function of the unknown parameter $\theta$, thus it also unknown. Then we need give an estimator of $R(\theta, \delta)$. Under the squared error loss, Bayes estimator $\Phi\left(\delta_{B}\right)$ of $R\left(\theta, \delta_{B}\right)$ is just the posterior mean of $R\left(\theta, \delta_{B}\right)$, then

$$
\begin{array}{r}
\Phi\left(\delta_{B}\right)=E\left[R\left(\theta, \delta_{B}\right) \mid X\right] \\
=\frac{1}{\left(\frac{3}{2} n+\alpha-1\right)^{2}}\left[\frac{(\beta+T)^{2}\left(\frac{3}{2} n+(\alpha-1)^{2}\right)}{\left(\frac{3}{2} n+\alpha-1\right)\left(\frac{3}{2} n+\alpha-2\right)}\right. \\
\left.+\frac{2 \beta(1-\alpha)(\beta+T)}{\frac{3}{2} n+\alpha-1}+\beta^{2}\right]
\end{array}
$$

\section{Conservative Properties of Estimation for Loss and Risk Function}

For convenience, in the following discussion we always suppose $\alpha \geq 0, \beta \geq 0$ and $n>2$. In this section, we obtain some conditions conservative properties of estimators under the loss function (8).

Theorem 2. The estimator $\gamma_{B}(X)$ is conservative under the loss function (8) whenever one of the following conditions holds:

i) $\alpha=0, n=1$, 
ii) $\alpha=0,0 \leq \beta \leq C \theta$,

where $C=\frac{4+2 \sqrt{9 n^{2}-12 n-8}}{n-3}, n \geq 2$

iii) $0 \leq \alpha \leq 2, \beta=0$

Proof. To see

(i) note that from Theorem 1 ,

$$
\begin{aligned}
& E_{\theta} \gamma_{B}(X)=\frac{\frac{3}{2} n\left(\frac{3}{2} n+1\right) \theta^{2}+3 \beta n \theta+\beta^{2}}{\left(\frac{3}{2} n-1\right)^{2}\left(\frac{3}{2} n-2\right)}, \\
& R\left(\theta, \delta_{B}\right)=\frac{\left(\frac{3}{2} n+1\right) \theta^{2}+2 \beta \theta+\beta^{2}}{\left(\frac{3}{2} n-1\right)^{2}}
\end{aligned}
$$

To show that $\gamma_{B}(x)$ is a conservative estimator, we need prove $E_{\theta} \gamma_{B}(X) \geq R\left(\theta, \delta_{B}\right)$.

Substitute (15) and (16) in the inequality:

$$
\left(\frac{3}{2} n-3\right) \beta^{2}-4 \beta \theta-2\left(\frac{3}{2} n+1\right) \theta^{2} \leq 0
$$

Obviously, if $n=1$, the inequality (17) holds. So the case (i) is proved.

When $n \geq 2,\{\beta \mid 0 \leq \beta \leq C \theta\}$ is the solution of (16), so the case (ii) also be proved.

For the case (iii), when $\beta=0$, from Theorem 1, we have

$$
\begin{aligned}
& E_{\theta} \gamma_{B}(X)=\frac{\frac{3}{2} n\left(\frac{3}{2} n+1\right) \theta^{2}}{\left(\frac{3}{2} n+\alpha-1\right)^{2}\left(\frac{3}{2} n+\alpha-2\right)}, \\
& R\left(\theta, \delta_{B}\right)=\frac{\left[\frac{3}{2} n+(\alpha-1)^{2}\right] \theta^{2}}{\left(\frac{3}{2} n+\alpha-1\right)^{2}}
\end{aligned}
$$

To show that $\gamma_{B}(x)$ is a conservative estimator, we need prove $E_{\theta} \gamma_{B}(X) \geq R\left(\theta, \delta_{B}\right)$.

Substitute (18) and (19) in the inequality, then we have:

$$
(\alpha-2)\left[\alpha^{2}+\left(\frac{3}{2} n-2\right) \alpha+\left(\frac{3}{2} n+1\right)\right] \leq 0
$$

We note that the former inequality always holds where $0 \leq \alpha \leq 2$, so the case (iii) is obtained.

Theorem 3 The estimator $\Phi\left(\delta_{B}\right)$ is conservative under the loss function (8) whenever one of the following conditions holds: (ii) $\alpha=0, \beta \geq 0$

(iii) $0 \leq \alpha \leq 2, \beta=0$

Proof. To see (i), when $\alpha=0$, from (13) we have

$$
E_{\theta} \Phi\left(\delta_{B}\right)=\frac{\frac{3}{2} n\left(\frac{3}{2} n+1\right)^{2} \theta^{2}+3 \beta n \theta(3 n-1)+\left(\frac{9}{4} n^{2}-1\right) \beta^{2}}{\left(\frac{3}{2} n-1\right)^{3}\left(\frac{3}{2} n-2\right)}
$$

To show that $\gamma_{B}(x)$ is a conservative estimator, we need prove $E_{\theta} \Phi\left(\delta_{B}\right) \geq R\left(\theta, \delta_{B}\right)$.

Substitute (12) and (20) in the inequality and simplify it, we have:

$$
\begin{aligned}
& 2\left(\frac{3}{2} n+1\right)(3 n-1) \theta^{2}+2\left(\frac{9}{4} n^{2}+3 n-2\right) \beta \theta \\
& +2\left(\frac{3}{2} n-1\right) \beta^{2} \geq 0
\end{aligned}
$$

We note that the former inquality always holds where $\beta \geq 0$, so the case (i) is obtained.

For the case (ii) when $\beta=0$, from (14) we have:

$$
\begin{aligned}
& \Phi\left(\delta_{B}\right)=\frac{T^{2}\left[\frac{3}{2} n+(\alpha-1)^{2}\right]}{\left(\frac{3}{2} n+\alpha-1\right)^{3}\left(\frac{3}{2} n+\alpha-2\right)} \\
& E_{\theta} \Phi\left(\delta_{B}\right)=\frac{\frac{3}{2} n\left(\frac{3}{2} n+1\right)\left[\frac{3}{2} n+(\alpha-1)^{2}\right] \theta^{2}}{\left(\frac{3}{2} n+\alpha-1\right)^{3}\left(\frac{3}{2} n+\alpha-2\right)}
\end{aligned}
$$

To show that $\gamma_{B}(x)$ is a conservative estimator, we need prove $E_{\theta} \Phi\left(\delta_{B}\right) \geq R\left(\theta, \delta_{B}\right)$.

Substitute (12) and (22) in the former inequality and simplification it, we have:

$$
(\alpha-2)(\alpha+3 n-1) \leq 0
$$

We note that it is always holds where $0 \leq \alpha \leq 2$, so (ii) is obtained.

\section{Conclusion}

This paper studies the Bayesian estimation of loss and risk functions Maxwell distribution under Rukhin's loss function. The conditions of conservative estimators are also discussed. According to the former discussion and considering the convenience of estimator $\gamma_{B}(X)$, for the estimation of loss function $w(\theta, \delta)$, we can get conclusions as following:

(i) If the prior parameter $\beta=0$, then $\gamma_{B}(X)$ is more reasonable; 
(ii) If the prior parameter $\beta \neq 0$, then when $\beta$ is a smaller number, we use $\gamma_{B}(X)$, otherwise we propose $\Phi\left(\delta_{B}\right)$;

(iii) Especially, if the prior distribution of $\theta$ is noninformative prior distribution (i.e. $\alpha=0, \beta=0$ ), or the matching prior distribution (i.e. $\alpha=1, \beta=0$ ), then the estimators $\gamma_{B}(X)$ and $\Phi\left(\delta_{B}\right)$ are both conservative estimators, but $\gamma_{B}(X)$ is more reasonable because of its simplicity.

\section{Acknowledgment}

This study is partially supported by Social Science Foundation of Hunan Province (No. 2015YBA065). The author also gratefully acknowledges the helpful comments and suggestions of the reviewers, which have improved the presentation.

\section{Conflict of Interest}

The author has declared that no conflict of interests exists.

\section{References}

[1] Rukhin A. L., 1988. Estimating the loss of estimators of binomial parameter. Biometrika, 75 (1): 153-155.

[2] Hu G., Li Q., Yu S., 2014. Optimal and minimax prediction in multivariate normal populations under a balanced loss function. Journal of Multivariate Analysis, 128 (14): 154-164.

[3] Cao L., Tao J., Shi N. Z. and Liu, W., 2015. A stepwise confidence interval procedure under unknown variances based on an asymmetric loss function for toxicological evaluation. Australian \& New Zealand Journal of Statistics, 57 (1), 73-98.

[4] Zakerzadeh H. and Zahraie S. H. M., 2014. Admissibility in non-regular family under squared-log error loss. Metrika, 78 (2): $227-236$.

[5] Ahmed E. A., 2014. Bayesian estimation based on progressive
Type-II censoring from two-parameter bathtub-shaped lifetime model: an Markov chain Monte Carlo approach. Journal of Applied Statistics, 41 (41): 752-768.

[6] Xu M. P. and Xiong L. C., 2009. Bayes inference for the loss and risk function in Levy distribution parameter estimation. Mathematics in Practice \& Theory, 39 (20): 221-226.

[7] Xian Z. H., 1993. Bayes inference for loss function. Mathematical Statistics and Applied Probability, 8 (3): 25-30.

[8] Xia Y. F. and Ma S. L., 2008. Bayes inference of loss and risk function in logarithmic normal distribution parameter estimation. Journal of Lanzhou University of Technology, 34 (1): 131-133.

[9] Ding X. Y. and Xu M. P., 2014. The Bayes inference for the loss and risk functions of parameters in normal and lognormal distribution. Journal of Jiangxi Normal University (Natural Sciences Edition), 38 (1): 70-73.

[10] Xu M. P., Ding X. Y. and Yu J., 2013. Bayes inference for the loss and risk functions of Rayleigh distribution parameter estimator. Mathematics in Practice \& Theory, 43 (21): 151156.

[11] Tyagi, R. K. and Bhattacharya S. K., 1989. Bayes estimation of the Maxwell's velocity distribution function, Statistica, 29 (4): $563-567$.

[12] Chaturvedi, A. and Rani U., 1998. Classical and Bayesian reliability estimation of the generalized Maxwell failure distribution, Journal of Statistical Research, 32: 113-120.

[13] Podder C. K. and Roy M. K., 2003. Bayesian estimation of the parameter of Maxwell distribution under MLINEX loss function. Journal of Statistical Studies, 23: 11-16.

[14] Bekker, A. and Roux J. J., 2005. Reliability characteristics of the Maxwell distribution: a Bayes estimation study, Comm. Stat. Theory \& Meth., 34 (11): 2169-2178.

[15] Dey S. and Sudhansu S. M., 2010. Bayesian estimation of the parameter of Maxwell distribution under different loss functions. Journal of Statistical Theory \& Practice, 4 (2): 279287.

[16] Krishna H. and Malik M., 2011. Reliability estimation in Maxwell distribution with progressively Type-II censored data. Journal of Statistical Computation \& Simulation, 82 (4): $1-19$. 